\title{
Histopathology of Facial Paraffinoma
}

Chi-Hsuan Chiang ${ }^{1}$ and Feng-Jie Lai ${ }^{1,2^{*}}$

${ }^{1}$ Department of Dermatology, Chi Mei Medical Center, Taiwan

${ }^{2}$ Center for General Education, Southern Taiwan University of Science and Technology, Taiwan

*Corresponding author: Feng-Jie Lai, Department of Dermatology, Chi Mei Medical Center, No.901, Zhonghua Rd., Yongkang Dist., Tainan City 710, Taiwan (R.O.C.), Tel: 886-6-2812811; extn. 57109; Fax: 886-6-2203706; E-mail: lai.fengjie@gmail.com

Rec date: Nov 22, 2014, Acc date: Nov 24, 2014, Pub date: Nov 26, 2014

Copyright: $\odot 2014$ Chiang $\mathrm{CH}$, et al. This is an open-access article distributed under the terms of the Creative Commons Attribution License, which permits unrestricted use, distribution, and reproduction in any medium, provided the original author and source are credited.

\section{Description}

Paraffinoma (sclerosing lipogranuloma) is characterized by a granulomatous inflammation of the skin due to introduction of mineral oils. We encountered a 61-year-old Taiwanese woman who received cosmetic local injection of unknown agents for facial augmentation 30 years ago in Indonesia. She noticed multiple asymptomatic indurated plaques on the forehead, nasal bridge, bilateral cheeks and chin (Figure 1) 2 weeks prior to visiting our department. The skin biopsy showed dense lymphohistiocytic infiltrate with multinucleated giant cell involving the entire dermis and epithelioid granulomas without caseous necrosis (Figure 2A).

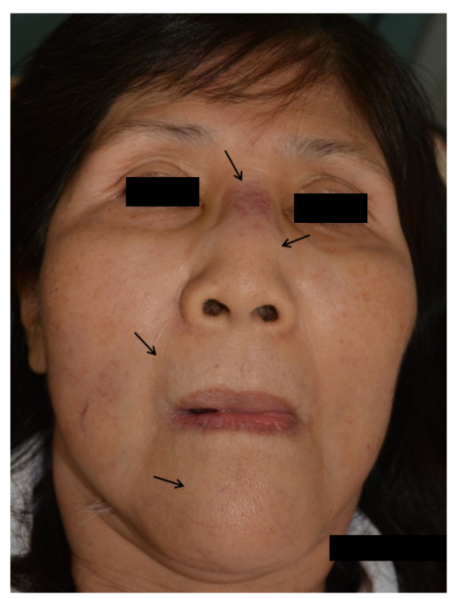

Figure 1: A 61-year-old woman presented to our department with multiple asymptomatic indurated plaques on the forehead, nasal bridge, bilateral cheeks and chin (arrows) 2 weeks prior to the visit. Notice the telangiectasia on the widened nasal bridge.

Variable-sized empty vacuoles and cysts in the dermis were reminiscent of Swiss cheese pattern (Figure 2B). The larger cysts lined by concentric fibrotic stroma, called onion-skin pattern was also noted (Figure 2C). Nile red staining confirmed a large number of lipid droplets in the dermis and subcutis (Figure 2D). It's difficult to differentiate paraffinoma from siliconoma in H\&E stain of histopathology because Swiss cheese and onion-skin pattern appeared in both diseases. Special stain of oil red O, Sudan IV stain can demonstrate the presence of oil in the variable-sized cysts. In our case, we used more sensitive fat stain of Nile red to enhance the interstitial small droplets in the dermis and subcutis, which can exclude silicone granuloma. The patient then received oral minocycline $100 \mathrm{mg}$ daily for the treatment of paraffinoma. The infiltrated plaques on the cheek and widened nasal bridge with prominent telangiectasia resolved dramatically after 2 week-therapy of minocycline.

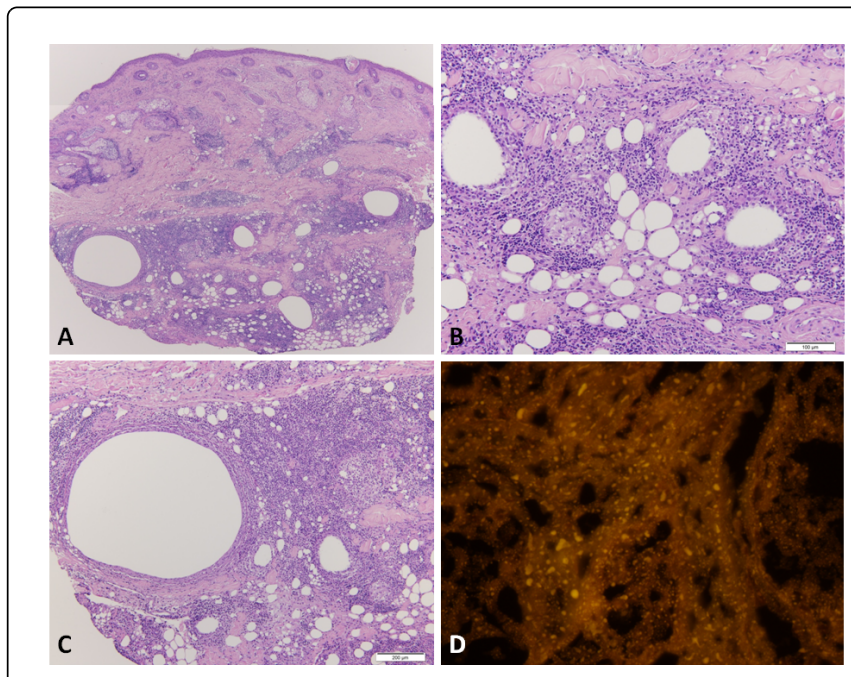

Figure 2: (A) The skin biopsy showed dense lymphohistiocytic infiltrate with multinucleated giant cell involving the entire dermis and epithelioid granulomas without caseous necrosis. (H\&E, 40X) (B) Variable-sized empty vacuoles and cysts in the dermis were reminiscent of Swiss cheese pattern. (H\&E, 200X) (C) The larger cysts lined by concentric fibrotic stroma, called onion-skin pattern was also noted. (H\&E, 100X) (D) Nile red staining demonstrated small lipid droplets in dermis and subcutis. (Nile red staining, 200X). 